\title{
Neurological manifestations as prognostic factors in COVID-19: a retrospective cohort study
}

\author{
Pedro Augusto Sampaio Rocha-Filho 1,2@ - João Eudes Magalhães ${ }^{2,4}$. Djanino Fernandes Silva² \\ Miriam Carvalho Soares ${ }^{3}$. Lucas Marenga Arruda Buarque ${ }^{3}$. Mylana Dandara Pereira Gama ${ }^{2}$. \\ Felipe Araújo Andrade Oliveira ${ }^{1,5}$
}

Received: 15 October 2021 / Accepted: 9 December 2021 / Published online: 21 January 2022

(c) Belgian Neurological Society 2021

\begin{abstract}
Background Neurological manifestations are frequent during COVID-19 but have been poorly studied as prognostic markers of COVID-19.

Objectives The aim of this study was to assess whether neurological manifestations are associated with a poor prognosis of COVID-19, and which patient and COVID-19 characteristics were associated with encephalopathy.

Methods This was a retrospective cohort study and included patients admitted with COVID-19 in four hospitals from Recife, Brazil. Data were collected by reviewing medical records.

Results 613 were included; $54.6 \%$ were male, the median age was 54 (41-68) years, 26.4\% required mechanical ventilation, and $24.1 \%$ died. The neurological symptoms presented were: myalgia $(25.6 \%)$, headache $(22 \%)$, fatigue $(22 \%)$, drowsiness $(16 \%)$, anosmia (14\%), disorientation $(8.8 \%)$, ageusia (7.3\%), seizures $(2.8 \%)$, and dizziness $(1.5 \%)$. Twelve patients $(2 \%)$ had strokes (ischemic strokes: 9) and 149 (24.3\%), encephalopathy. Older age, a prolonged hospitalization, diabetes mellitus, a previous history of stroke and having epileptic seizures during hospitalization were significantly associated with the occurrence of encephalopathy. Older age, smoking and requiring mechanical ventilation were associated with prolonged hospitalization. Older patients, those requiring mechanical ventilation and those with encephalopathy presented a significantly higher risk, while those who had anosmia presented a significantly lower risk of dying.

Conclusions Neurological symptoms are frequent among patients with COVID-19. Encephalopathy was the most frequent neurological complication and was associated with a higher mortality. Those with anosmia had a lower mortality.
\end{abstract}

Keywords Neurologic manifestations $\cdot$ Neurologic symptoms $\cdot$ COVID-19 $\cdot$ Encephalopathy $\cdot$ Mortality $\cdot$ Prognosis

\section{Introduction}

Pedro Augusto Sampaio Rocha-Filho

pasrf1@hotmail.com; pedroasampaio@gmail.com

1 Division of Neuropsychiatry, Centro de Ciências Médicas, Universidade Federal de Pernambuco (UFPE), Rua General Joaquim Inacio, 830, Sala 1412, Edf The Plaza Business Center, Recife, PE CEP: 50070-495, Brazil

2 Hospital Universitario Oswaldo Cruz, Universidade de Pernambuco (UPE), Recife, Brazil

3 Hospital das Clínicas (HC-UFPE), Recife, Brazil

4 Instituto de Medicina Integral Professor Fernando Figueira (IMIP), Recife, Brazil

5 Real Hospital Português de Beneficência de Pernambuco, Recife, Brazil
Neurological symptoms are frequent during COVID-19, and occur in $8-92 \%$ of hospitalized patients [1-5]. These include headache, anosmia, ageusia, myalgia, dizziness, fatigue, altered level of consciousness, mental confusion, agitation, and epileptic seizures. These symptoms are among those that most trouble patients during the course of the disease. Although respiratory symptoms are the main cause of hospitalization in COVID-19, neurological symptoms may also lead patients to seek hospital care [4].

Headache, anosmia and ageusia are symptoms that generally occur in patients with a milder form of the disease at the onset of clinical symptoms, and often occur grouped together [4, 6-13], while mental confusion, agitation and epileptic seizures are more frequent in more severe cases 
of the disease $[4,11,14,15]$. Neurological symptoms have been poorly studied as prognostic markers of COVID- 19 .

While neurological complications are rare in terms of population [16], they are not infrequent among hospitalized patients, and are even more frequent in severe cases of COVID-19 [3, 12, 17-19]. The neurological complications described include cerebrovascular diseases, encephalopathies, encephalitis and meningitis, acute disseminated encephalomyelitis, myelitis, Guillain-Barré syndrome, multiple demyelinating sensory and motor mononeuropathy, and myopathies [3-5, 12, 18-24]. An Italian study that compared patients with and without COVID-19 admitted to a neurological unit with neurological complications reported mortality and length of hospital stay significantly higher among those with COVID-19. [25]

Several mechanisms may be involved in the genesis of symptoms and neurological complications in COVID-19. These include direct viral damage, a systemic inflammatory response, hypoxia, drugs used in patient management, and vascular complications [26]. Since there are different underlying mechanisms, our hypothesis is that different neurological manifestations may be used as prognostic markers of different risks.

The aim of this study was to assess whether neurological manifestations are associated with a poor prognosis of COVID-19 in hospitalized patients, and which patient and COVID-19 characteristics were associated with encephalopathy.

\section{Methods}

This was a retrospective cohort study conducted in the city of Recife, state capital of Pernambuco, located in Northeast Brazil, and involved four hospitals:

1. Hospital Universitário Oswaldo Cruz (HUOC-UPE). This hospital is linked to the Universidade de Pernambuco and attends patients from the public health system.

2. Hospital das Clínicas (HC-UFPE). This hospital is linked to the Universidade Federal de Pernambuco and attends patients from the public health system.

3. Instituto de Medicina Integral Professor Fernando Figueira (IMIP). This hospital attends patients from the public system and is linked to the Faculdade Pernambucana de Saúde.

4. Real Hospital Português de Beneficência de Pernambuco (RHP). This hospital attends patients from the private sector, generally with private health insurance.

Patients included in the study were those of both sexes, admitted with COVID-19 in April and May 2020, confirmed by reverse transcription polymerase chain reaction
(RT-PCR) technique, with material collected from nasal and oropharynx swabs. Pregnant women and those aged under six years were excluded.

Data were collected by neurologists and neurology residents by reviewing medical records. For this, a specific form was developed, which contained: sociodemographic data; clinical data, such as the presence of comorbidities, laboratory tests performed, signs and symptoms presented, and the need for mechanical ventilation; the need for admission to the intensive care unit (ICU), the presence of neurological symptoms, such as dizziness, fatigue, headache, anosmia, ageusia, myalgia, seizures, altered level of consciousness and mental confusion; the presence of neurological complications; the occurrence of death and the days of hospitalization.

Patients presenting with a disturbance in attention and awareness (reduced orientation to the environment) developed over a short period and represented a change from baseline were considered to have delirium. [27]

Patients presenting with delirium and/or a decreased level of consciousness, which were not associated with the occurrence of stroke or another specific neurological diagnosis that justified these complaints were considered to have encephalopathy. [27]

Rhabdomyolysis was considered when the highest level of creatine kinase (CK) was greater than the 75 th percentile for the sample.

A prolonged hospitalization was defined as a time greater than or equal to the 75 th percentile.

Older patients were considered as those aged over 60 years.

\section{Statistical analysis}

The statistical analyses were performed using SPSS Statistics Software version 21.0 (IBM Corporation, Armonk, NY, USA).

Quantitative data were tested regarding normality of distribution, by the means of the Kolmogorov-Smirnov test. When the distribution was normal, the means and standard deviations were calculated. If not, the medians and the 25 th and 75th percentiles were used $\left(\mathrm{P}_{25} ; \mathrm{P}_{75}\right)$.

The percentage distribution of the categorical variables was compared between the groups by means of the Chisquare test or Fisher's exact test. Numerical variables were compared using the Mann-Whitney test.

For the analyzes referring to death and length of hospital stay, if the patient presented with a neurological symptom that could be explained by a specific neurological diagnosis, the specific neurological diagnosis was used in the analysis.

Logistic regression models were used to assess what is associated with the presence of encephalopathy and whether neurological symptoms or complications were associated 
with mortality and a prolonged hospitalization. Variables that presented some association with these prognostic factors in the univariate analysis $(P$ value $<0.05)$ were initially included in the model using the stepwise method. Only those variables that caused no loss of stability remained in the model.

All tests were leveled by a 0.05 significance.

\section{Ethical aspects}

The research was approved by the Research Ethics Committees of the hospitals involved. Since data was collected through medical records, the informed consent form was waived by the Ethics Committees.

\section{Results}

A total of 701 medical records were reviewed, 82 patients were excluded for being pregnant and six for being aged under six years. Six hundred and thirteen patients were considered for analysis, $35.7 \%$ were patients from HUOC-UPE; $27.6 \%$, from RHP; $22.7 \%$, from IMIP; and $14 \%$, from HCUFPE. Three hundred and thirty-five (54.6\%) were male, the median age was 54 (41-68) years, 248 (40.5\%) were older patients; 239 patients (39\%) needed to be admitted to ICU and $162(26.4 \%)$ required mechanical ventilation. The median length of time on mechanical ventilation was 8 days (4-14).

These patients confirmed previous diseases: systemic arterial hypertension (47\%), diabetes mellitus (29\%), obesity (14.2\%), smoking (8.5\%), chronic kidney disease (8.3\%), cancer (7.5\%), stroke (2.9\%); epilepsy (1.1\%) and other diseases $(<5 \%)$.
Symptoms presented during COVID-19 were cough (83\%), fever (79\%), dyspnea (73\%), diarrhea (14\%) and runny nose $(11 \%)$. Asthenia, chills, sore throat, joint pain, chest pain, abdominal pain, nausea or vomiting were reported by less than $10 \%$ of patients. The first pulse oximetry was recorded for 552 patients and presented a median of 95 (91-97\%), but 229 patients $(41.5 \%)$ presented $\mathrm{SpO}_{2}<94 \%$ and 100 patients (18.1\%), $\mathrm{SpO}_{2}<90 \%$.

The neurological symptoms presented during COVID19 were myalgia $(25.6 \%)$, headache $(22 \%)$, fatigue $(22 \%)$, drowsiness (16\%), anosmia (14\%), disorientation (8.8\%), ageusia (7.3\%), and dizziness (1.5\%).

Seventeen patients $(2.8 \%)$ presented epileptic seizures, one of whom underwent cerebral magnetic resonance, cerebral magnetic resonance angiogram and cerebrospinal fluid test, which were normal. One of these patients had a previous history of epilepsy.

Twelve patients (2\%) had strokes during COVID-19, of which, 9 were ischemic strokes and 3 were hemorrhagic. All patients with stroke were aged over 60 years, seven had hypertension, five had diabetes mellitus, one was a smoker, one had atrial fibrillation and one had heart failure. Five of those with stroke had suffered previous strokes.

Serum CK levels were recorded in 345 (56\%) patients, which presented a median of 218.5 (73-516.5). Eighty of these patients (23.2\%) presented a CK above the 75th percentile, indicating rhabdomyolysis.

One hundred and forty-nine (24.3\%) patients presented encephalopathy. Table 1 demonstrates the laboratory profile of patients with encephalopathy. Those with encephalopathy presented significantly higher levels of neutrophils, C-reactive protein, urea, creatinin, aspartate aminotransferase and lactate dehydrogenase, and lower levels of hemoglobin and CK than those without encephalopathy.

Table 1 The laboratory data of those with and without encephalopathy

\begin{tabular}{|c|c|c|c|c|c|}
\hline Laboratory tests & $N$ & Total (median) & $\begin{array}{l}\text { With encephalopathy } \\
\text { (median) }\end{array}$ & $\begin{array}{l}\text { Without encephalopa- } \\
\text { thy (median) }\end{array}$ & $P$ value \\
\hline Hemoglobin (g/dL) & 589 & $13.5(11.4-14.4)$ & $12.3(9.8-14.8)$ & $13.5(13-14)$ & $<0.01$ \\
\hline Neutrophil count $\left(10^{3} / \mathrm{mcL}\right)$ & 581 & $6.47(4.83-9.15)$ & $9.15(6.9-11.4)$ & $4.83(3.61-6.05)$ & $<0.01$ \\
\hline Lymphocyte count $\left(10^{3} / \mathrm{mcL}\right)$ & 581 & $1.27(1.05-1.43)$ & $1.27(1.24-1.3)$ & $1.21(0.85-1.57)$ & 0.24 \\
\hline Platelets $\left(10^{3} / \mathrm{mcL}\right)$ & 581 & $217.5(171.5-430.5)$ & $385(172-598)$ & $217(171-263)$ & 0.60 \\
\hline C-Reactive protein (mg/dL) & 515 & $70.5(16.9-156.5)$ & $156.5(114-149)$ & $16.9(6.9-27)$ & $<0.01$ \\
\hline D-Dimer (mcg/mL) & 204 & $212.8(3.6-496)$ & $3.55(1.6-5.5)$ & $496(420-572)$ & 0.23 \\
\hline Urea (mg/dL) & 574 & $40.5(28-96)$ & $90.5(35-146)$ & $33.5(21-46)$ & $<0.01$ \\
\hline Creatinin (mg/dL) & 579 & $1.09(0.85-2.09)$ & $1.85(0.7-3)$ & $1.08(1-1.17)$ & $<0.01$ \\
\hline Aspartate aminotransferase (U/L) & 541 & $47.5(34.5-73.5)$ & $73.5(55-92)$ & $34.5(29-40)$ & $<0.01$ \\
\hline Alanine aminotransferase (U/L) & 541 & $32.5(27.5-44.5)$ & $44.5(35-54)$ & $27.5(25-30)$ & 0.94 \\
\hline Lactate dehydrogenase (U/L) & 422 & $513(316.5-693.5)$ & $513(436-590)$ & 497 (197-797) & $<0.01$ \\
\hline Ferritin $\left(10^{3} \mathrm{ng} / \mathrm{mL}\right)$ & 371 & $1.39(1.2-1.71)$ & $1.68(1.36-2)$ & $1.23(1.04-1.42)$ & 0.24 \\
\hline Creatinine kinase (U/L) & 345 & $218.5(73-516.5)$ & $205.5(60-351)$ & $384(86-682)$ & $<0.01$ \\
\hline
\end{tabular}


Table 2 presents the sociodemographic and clinical characteristics which were associated with encephalopathy. After controlling for the variables of confusion, older age, a prolonged hospitalization, diabetes mellitus, a previous history of stroke and having epileptic seizures during hospitalization were significantly associated with the occurrence of encephalopathy.

The median length of hospital stay was 7 (4-13) days. One hundred and forty-two patients had prolonged hospitalization. Table 3 demonstrates that sociodemographic and clinical characteristics were associated with prolonged hospitalization. Older age, smoking, and requiring mechanical ventilation were associated with prolonged hospitalization. After repeating this analysis without the patients who had died, there was no change in these results (data not shown).

There were 148/613 (24.1\%) hospital deaths. The variables associated with deaths are described in Table 4. After controlling for the confounding variables, older patients, and those requiring mechanical ventilation and with encephalopathy presented a significantly higher risk, while those who had anosmia presented a significantly lower risk of dying.

Table 2 Estimated risk for encephalopathy according to patient sociodemographic and clinical characteristics

\begin{tabular}{|c|c|c|c|c|c|c|}
\hline Characteristics & Total $(N=613)$ & $\begin{array}{l}\text { With encepha- } \\
\text { lopathy }(N=149)\end{array}$ & $\begin{array}{l}\text { Without encepha- } \\
\text { lopathy }(N=464)\end{array}$ & RR (95\% CI) & $P$ value & Adjusted RR (95\% CI) \\
\hline Aged $>60$ years & $248(40.5)$ & $100(67.1)$ & $148(31.9)$ & $1.45(1.3-1.62)$ & $<0.01$ & $3.59(2.32-5.54)$ \\
\hline Male & $335(54.6)$ & $85(57)$ & $250(54)$ & $1.03(0.94-1.13)$ & 0.49 & - \\
\hline \multicolumn{7}{|l|}{ Comorbidity } \\
\hline Smoking & $52(8.5)$ & $13(8.7)$ & $39(8.4)$ & $1.01(0.86-1.19)$ & 0.9 & - \\
\hline Alcoholism & $13(2.1)$ & $3(2)$ & $10(2.2)$ & $0.98(0.73-1.33)$ & 0.93 & - \\
\hline Obesity & 87 (14.2) & $14(9.4)$ & $73(15.7)$ & $0.89(0.8-0.98)$ & 0.05 & - \\
\hline Hypertension & $288(47)$ & $90(60.4)$ & $198(42.7)$ & $1.19(1.08-1.31)$ & $<0.01$ & - \\
\hline Diabetes mellitus & $178(29)$ & $68(45.6)$ & $110(23.7)$ & $1.32(1.16-1.49)$ & $<0.01$ & $1.95(1.26-3.02)$ \\
\hline Ischemic heart disease & $27(4.4)$ & $10(6.7)$ & $17(3.7)$ & $1.21(0.9-1.62)$ & 0.12 & - \\
\hline Heart failure & $26(4.2)$ & $12(8.1)$ & $14(3)$ & $1.42(0.99-2.04)$ & $<0.01$ & - \\
\hline Valvular heart disease & $3(0.5)$ & $1(0.7)$ & $2(0.4)$ & $1.14(0.51-2.53)$ & 0.72 & - \\
\hline Atrial fibrillation & $16(2.6)$ & $8(5.4)$ & $8(1.7)$ & $1.53(0.93-2.5)$ & 0.02 & - \\
\hline Asthma & $29(4.7)$ & $2(1.3)$ & $27(5.8)$ & $0.8(0.72-0.9)$ & 0.03 & - \\
\hline $\begin{array}{l}\text { Chronic obstructive pulmo- } \\
\text { nary disease }\end{array}$ & $24(3.9)$ & $8(5.4)$ & $16(3.4)$ & $1.14(0.86-1.52)$ & 0.29 & - \\
\hline Chronic renal disease & $51(8.3)$ & $21(14.1)$ & $30(6.5)$ & $1.32(1.04-1.66)$ & $<0.01$ & - \\
\hline Chronic liver disease & $10(1.6)$ & $4(2.7)$ & $6(1.3)$ & $1.27(0.76-2.1)$ & 0.24 & - \\
\hline Prior stroke & $18(2.9)$ & $14(9.4)$ & $4(0.9)$ & $3.48(1.46-8.27)$ & $<0.01$ & $4.85(1.38-17.02)$ \\
\hline Epilepsy & $7(1.1)$ & $2(1.3)$ & $5(1.1)$ & $1.06(0.66-1.7)$ & 0.79 & - \\
\hline \multicolumn{7}{|l|}{ Clinical data } \\
\hline Fever & $482(78.6)$ & $108(72.5)$ & 374 (80.6) & $0.88(0.78-1.0)$ & 0.04 & - \\
\hline Dyspnoea & 445 (72.6) & $115(77.2)$ & $330(71.1)$ & $1.08(0.98-1.18)$ & 0.15 & - \\
\hline Mechanical ventilation & $162(26.4)$ & $84(56.4)$ & $78(16.8)$ & $1.78(1.51-2.1)$ & $<0.01$ & - \\
\hline Seizure & $17(2.8)$ & $15(10.1)$ & $2(0.4)$ & $6.59(1.79-24.2)$ & $<0.01$ & $37.3(7.9-176.02)$ \\
\hline Fatigue & $135(22)$ & $24(16.1)$ & $111(23.9)$ & $0.9(0.82-0.98)$ & 0.04 & - \\
\hline Anosmia & $86(14)$ & $5(3.4)$ & $81(17.5)$ & $0.77(0.72-0.83)$ & $<0.01$ & - \\
\hline Ageusia & $45(7.3)$ & $2(1.3)$ & $43(9.3)$ & $0.78(0.72-0.84)$ & 0.01 & - \\
\hline Headache & $135(22)$ & $11(7.4)$ & $124(26.7)$ & $0.77(0.72-0.84)$ & $<0.01$ & - \\
\hline Myalgia & $157(25.6)$ & $16(10.7)$ & $141(30.4)$ & $0.79(0.73-0.85)$ & $<0.01$ & - \\
\hline Dizziness & $9(1.5)$ & $3(2)$ & $6(1.3)$ & $1.14(0.71-1.81)$ & 0.52 & - \\
\hline Stroke & $12(2)$ & $9(6)$ & $3(0.6)$ & $3.07(1.15-8.18)$ & $<0.01$ & - \\
\hline Ischemic stroke & $9(1.5)$ & $7(4.7)$ & $2(0.4)$ & $3.44(1.01-11.7)$ & $<0.01$ & - \\
\hline Hemorrhagic stroke & $3(0.5)$ & $2(1.3)$ & $1(0.2)$ & $2.28(0.46-11.3)$ & 0.08 & - \\
\hline Prolonged hospitalization & $142(23)$ & $59(39.6)$ & $83(17.9)$ & $1.38(1.19-1.6)$ & $<0.01$ & $2.17(1.38-3.41)$ \\
\hline
\end{tabular}

$R R$ relative risk 
Table 3 Estimated risk for prolonged hospitalization according to patient sociodemographic and clinical characteristics

\begin{tabular}{|c|c|c|c|c|c|c|}
\hline Characteristics & Total $(N=613)$ & $\begin{array}{l}\text { Length of hospital } \\
\text { stay }(>13 \text { days }) \\
(N=142)\end{array}$ & $\begin{array}{l}\text { Length of hospital } \\
\text { stay }(<14 \text { days }) \\
(N=471)\end{array}$ & RR (95\% CI) & $P$ value & Adjusted RR (95\% CI) \\
\hline Aged $>60$ years & $248(40.5)$ & $81(57)$ & $167(35.5)$ & $1.24(1.12-1.36)$ & $<0.01$ & $1.58(1.03-2.43)$ \\
\hline Male & $335(54.6)$ & $79(55.6)$ & $256(54.4)$ & $1.01(0.93-1.1)$ & 0.79 & - \\
\hline \multicolumn{7}{|l|}{ Comorbidity } \\
\hline Smoking & $52(8.5)$ & $20(14.1)$ & $32(6.8)$ & $1.27(1.02-1.58)$ & $<0.01$ & $2.11(1.1-4.05)$ \\
\hline Alcoholism & $13(2.1)$ & $4(2.8)$ & $9(1.9)$ & $1.11(0.77-1.6)$ & 0.51 & - \\
\hline Obesity & $87(14.2)$ & $20(14.1)$ & $67(14.2)$ & $0.99(0.88-1.13)$ & 0.97 & - \\
\hline Hypertension & $288(47)$ & $81(57)$ & $207(44)$ & $1.13(1.03-1.24)$ & $<0.01$ & - \\
\hline Diabetes mellitus & $178(29)$ & $59(41.5)$ & $119(25.3)$ & $1.21(1.08-1.35)$ & $<0.01$ & - \\
\hline Ischemic heart disease & $27(4.4)$ & $9(6.3)$ & $18(3.8)$ & $1.16(0.88-1.52)$ & 0.20 & - \\
\hline Heart failure & $26(4.2)$ & $13(9.2)$ & $13(2.8)$ & $1.56(1.06-2.29)$ & $<0.01$ & - \\
\hline Valvular heart disease & $3(0.5)$ & $1(0.7)$ & $2(0.4)$ & $1.15(0.52-2.57)$ & 0.68 & - \\
\hline Atrial fibrillation & $16(2.6)$ & $6(4.2)$ & $10(2.1)$ & $1.24(0.84-1.81)$ & 0.17 & - \\
\hline Asthma & $29(4.7)$ & $4(2.8)$ & $25(5.3)$ & $0.89(0.76-1.03)$ & 0.22 & - \\
\hline $\begin{array}{l}\text { Chronic obstructive } \\
\text { pulmonary disease }\end{array}$ & $24(3.9)$ & $8(5.6)$ & $16(3.4)$ & $1.16(0.87-1.54)$ & 0.23 & - \\
\hline Chronic renal disease & $51(8.3)$ & $19(13.4)$ & $32(6.8)$ & $1.24(1.0-1.54)$ & 0.01 & - \\
\hline Chronic liver disease & $10(1.6)$ & $4(2.8)$ & $6(1.3)$ & $1.28(0.77-2.14)$ & 0.20 & - \\
\hline Prior stroke & $18(2.9)$ & $9(6.3)$ & $9(1.9)$ & $1.55(0.98-2.47)$ & $<0.01$ & - \\
\hline Epilepsy & $7(1.1)$ & - & $7(1.5)$ & - & - & - \\
\hline \multicolumn{7}{|l|}{ Clinical data } \\
\hline Fever & $482(78.6)$ & $113(79.6)$ & $369(78.3)$ & $1.02(0.92-1.13)$ & 0.75 & - \\
\hline Dyspnoea & 445 (72.6) & $107(75.4)$ & $38(71.8)$ & $1.04(0.95-1.14)$ & 0.40 & - \\
\hline Mechanical ventilation & $162(26.4)$ & $70(49.3)$ & $92(19.5)$ & $1.48(1.29-1.7)$ & $<0.01$ & $3.58(2.34-5.48)$ \\
\hline $\begin{array}{l}\text { Decreased level of } \\
\text { consciousness }\end{array}$ & $98(16)$ & $43(30.3)$ & $61(13)$ & $1.37(1.16-1.62)$ & $<0.01$ & - \\
\hline Disorientation & $54(8.8)$ & $26(18.3)$ & $30(6.4)$ & $1.48(1.15-1.89)$ & $<0.01$ & - \\
\hline Encephalopathy & $149(24.3)$ & $59(41.5)$ & $90(19.1)$ & $1.36(1.19-1.56)$ & $<0.01$ & - \\
\hline Seizure & $17(2.8)$ & $6(4.2)$ & $11(2.3)$ & $1.19(0.84-1.7)$ & 0.23 & - \\
\hline Fatigue & $135(22)$ & $31(21.8)$ & $104(22.1)$ & $0.99(0.89-1.11)$ & 0.95 & - \\
\hline Anosmia & $86(14)$ & $12(8.5)$ & $74(15.7)$ & $0.87(0.79-0.97)$ & 0.03 & - \\
\hline Ageusia & $45(7.3)$ & $7(4.9)$ & $38(8.1)$ & $0.9(0.79-1.03)$ & 0.21 & - \\
\hline Headache & $135(22)$ & $23(16.2)$ & $112(23.8)$ & $0.91(0.83-1.0)$ & 0.05 & - \\
\hline Myalgia & $157(25.6)$ & $17(12)$ & $40(29.7)$ & $0.81(0.75-0.88)$ & $<0.01$ & - \\
\hline Dizziness & $9(1.5)$ & $3(2.1)$ & $6(1.3)$ & $1.15(0.73-1.84)$ & 0.47 & - \\
\hline Stroke & $12(2)$ & $6(4.2)$ & $6(1.3)$ & $1.55(0.88-2.73)$ & 0.03 & - \\
\hline Ischemic stroke & $9(1.5)$ & $4(2.8)$ & $5(1.1)$ & $1.39(0.77-2.49)$ & 0.13 & - \\
\hline Hemorrhagic stroke & $3(0.5)$ & $2(1.4)$ & $1(0.2)$ & $2.31(0.47-11.46)$ & 0.07 & - \\
\hline
\end{tabular}

$R R$ relative risk

\section{Discussion}

Our patients presented with myalgia, headache, fatigue, drowsiness, anosmia, disorientation, and ageusia as the most frequent neurological symptoms. This is in agreement with the literature $[1-5,11,12,18]$.

We observed a stroke incidence of $2 \%, 75 \%$ of which were ischemic strokes. Most stroke patients presented with risk factors for vascular disease and $42 \%$ had a previously history of stroke. The hospital series that assessed neurological complications of COVID-19 reported a stroke frequency similar to ours (from 1 to $3.3 \%$ ), with ischemic stroke being two to five times more frequent than hemorrhagic stroke $[1,3,5,11,12,18,28]$. A meta-analysis that included more than 100,000 patients with COVID-19 reported an incidence of cerebrovascular disease of $1.4 \%$ (1.0-1.9), with ischemic stroke being the most common 
Table 4 Estimated risk for death according to patient sociodemographic and clinical characteristics

\begin{tabular}{|c|c|c|c|c|c|c|}
\hline Characteristics & Total $(N=613)$ & $\begin{array}{l}\text { Death out- } \\
\text { come: yes } \\
(N=148)\end{array}$ & $\begin{array}{l}\text { Death } \\
\text { outcome: no } \\
(N=465)\end{array}$ & RR $(95 \%$ CI $)$ & $P$ value & Adjusted RR (95\% CI) \\
\hline Aged $>60$ years & $248(40.5)$ & $102(68.9)$ & $146(31.4)$ & $1.48(1.33-1.66)$ & $<0.01$ & $3.92(2.17-7.08)$ \\
\hline Male & $335(54.6)$ & $87(58.8)$ & $248(53.3)$ & $1.05(0.96-1.15)$ & 0.25 & - \\
\hline \multicolumn{7}{|l|}{ Comorbidity } \\
\hline Smoking & $52(8.5)$ & $13(8.8)$ & $39(8.4)$ & $1.01(0.86-1.19)$ & 0.88 & - \\
\hline Alcoholism & $13(2.1)$ & $4(2.7)$ & $9(1.9)$ & $1.09(0.76-1.58)$ & 0.57 & - \\
\hline Obesity & $87(14.2)$ & $24(16.2)$ & $63(13.5)$ & $1.05(0.92-1.21)$ & 0.42 & - \\
\hline Hypertension & $288(47)$ & $77(52)$ & $211(45.4)$ & $1.07(0.97-1.17)$ & 0.16 & - \\
\hline Diabetes mellitus & $178(29)$ & $57(38.5)$ & $121(26)$ & $1.16(1.04-1.3)$ & $<0.01$ & - \\
\hline Ischemic heart disease & $27(4.4)$ & $9(6.1)$ & $18(3.9)$ & $1.14(0.87-1.5)$ & 0.25 & - \\
\hline Heart failure & $26(4.2)$ & $12(8.1)$ & $14(3)$ & $1.43(1.0-2.04)$ & $<0.01$ & - \\
\hline Valvular heart disease & $3(0.5)$ & $2(1.4)$ & $1(0.2)$ & $2.28(0.46-11.3)$ & 0.08 & - \\
\hline Atrial fibrillation & $16(2.6)$ & $11(7.4)$ & $5(1.1)$ & $2.47(1.19-5.11)$ & $<0.01$ & - \\
\hline Asthma & $29(4.7)$ & $2(1.4)$ & $27(5.8)$ & $0.81(0.72-0.9)$ & 0.03 & - \\
\hline Chronic obstructive pulmonary disease & $24(3.9)$ & $7(4.7)$ & $17(3.7)$ & $1.07(0.83-1.39)$ & 0.56 & - \\
\hline Chronic renal disease & $51(8.3)$ & $26(17.6)$ & $25(5.4)$ & $1.6(1.2-2.12)$ & $<0.01$ & - \\
\hline Chronic liver disease & $10(1.6)$ & $3(2)$ & $7(1.5)$ & $1.09(0.72-1.63)$ & 0.66 & - \\
\hline Prior stroke & $18(2.9)$ & $8(5.4)$ & $10(2.2)$ & $1.38(0.91-2.09)$ & 0.04 & - \\
\hline Epilepsy & $7(1.1)$ & $1(0.7)$ & $6(1.3)$ & $0.88(0.65-1.2)$ & 0.54 & - \\
\hline \multicolumn{7}{|l|}{ Clinical data } \\
\hline Fever & $482(78.6)$ & $106(71.6)$ & $376(80.9)$ & $0.87(0.77-0.99)$ & 0.02 & - \\
\hline Dyspnoea & 445 (72.6) & $125(84.5)$ & $320(68.8)$ & $1.2(1.1-1.3)$ & $<0.01$ & - \\
\hline Mechanical ventilation & $162(26.4)$ & $117(79.1)$ & $45(9.7)$ & $3.35(2.61-4.3)$ & $<0.01$ & $27.4(15.2-49.5)$ \\
\hline Decreased level of consciousness & $98(16)$ & $59(39.9)$ & $39(8.4)$ & $2.06(1.62-2.61)$ & $<0.01$ & - \\
\hline Disorientation & $54(8.8)$ & $26(17.6)$ & $28(6)$ & $1.51(1.17-1.95)$ & $<0.01$ & - \\
\hline Encephalopathy & $149(24.3)$ & $83(56.1)$ & $66(14.2)$ & $1.94(1.62-2.33)$ & $<0.01$ & $2.7(1.51-4.83)$ \\
\hline Seizure & $17(2.8)$ & $8(5.4)$ & $9(1.9)$ & $1.44(0.92-2.27)$ & 0.03 & - \\
\hline Fatigue & $135(22)$ & $26(17.6)$ & $109(23.4)$ & $0.92(0.84-1.02)$ & 0.13 & - \\
\hline Anosmia & $86(14)$ & $2(1.4)$ & $84(18.1)$ & $0.74(0.7-0.79)$ & $<0.01$ & $0.17(0.03-0.81)$ \\
\hline Ageusia & $45(7.3)$ & $3(2)$ & $42(9)$ & $0.8(0.73-0.88)$ & $<0.01$ & - \\
\hline Headache & $135(22)$ & $13(8.8)$ & $122(26.2)$ & $0.79(0.73-0.86)$ & $<0.01$ & - \\
\hline Myalgia & $157(25.6)$ & $14(9.5)$ & $143(30.8)$ & $0.77(0.72-0.84)$ & $<0.01$ & - \\
\hline Dizziness & $9(1.5)$ & $1(0.7)$ & $8(1.7)$ & $0.85(0.67-1.08)$ & 0.36 & - \\
\hline Stroke & $12(2)$ & $5(3.4)$ & $7(1.5)$ & $1.31(0.81-2.11)$ & 0.15 & - \\
\hline Ischemic stroke & $9(1.5)$ & $3(2)$ & $6(1.3)$ & $1.14(0.72-1.81)$ & 0.52 & - \\
\hline Hemorrhagic Stroke & $3(0.5)$ & $2(1.4)$ & $1(0.2)$ & $2.28(0.46-11.3)$ & 0.08 & - \\
\hline Prolonged hospitalization & $142(23)$ & $43(29.1)$ & $99(21.3)$ & $1.12(0.99-1.26)$ & 0.04 & - \\
\hline
\end{tabular}

$R R$ relative risk

type. Those with stroke presented cardiovascular risk factors more often than those without stroke [29].

Almost a quarter of our patients who measured CK levels presented a significant increase in $\mathrm{CK}$, indicating muscle damage. We cannot rule out that patients with muscle symptoms have dosed this enzyme more frequently, and that these levels are overestimated. However, a meta-analysis that included more than two thousand patients reported that $17 \%$ (11-22\%) of the patients presented elevated levels of CK levels, which is close to ours. Elevated CK levels are associated with a threefold increase in the risk of a poor prognosis [30]. Since only $56 \%$ of patients measured CK levels, we decided not to include it in the prognostic analyses.

Encephalopathy was the most frequent neurological complication, occurring in $24 \%$ of our patients. The term encephalopathy refers to a brain dysfunction unexplained by any other neurological disease, which usually develops acutely and may have symptoms such as subsyndromal 
delirium, delirium, or a decreased level of consciousness [27]. Unlike encephalitis, encephalopathy is not caused by the direct action of viral invasion and is multifactorial. Contributing factors for its appearance are the occurrence of hypoxic-ischemic alterations, toxic and metabolic alterations, the use of drugs for the treatment of these patients, the inflammatory response secondary to sepsis, and the failure of multiple organs [31]. The frequency of encephalopathy in different studies with patients hospitalized with COVID-19 ranged from 10 to $60 \%[1,3-5,11,15,32]$, and was higher in those studies that included more critically ill patients [31].

Our patients with encephalopathy presented higher levels of neutrophils and C-reactive protein, and greater changes in the liver enzymes and renal function. This suggests that sepsis and metabolic changes are associated with the development of encephalopathy. According to other studies, other factors may also be associated with its genesis, such as hypoxia, drugs used in the treatment of these patients and ischemic alterations $[4,14,31]$.

Older patients, those with previous illnesses (diabetes mellitus and stroke), those who had epileptic seizures during hospitalization and those with prolonged hospitalization presented more encephalopathy. Liotta et al. reported a higher risk for encephalopathy in patients with COVID-19 who had some previous neurological disease, and encephalopathy was also associated with a longer length of hospital stay [3]. Other studies have reported that patients with COVID-19 who were older [15, 32] and with a higher number of chronic diseases [15] have a higher risk of delirium and older patients are more likely to have an altered level of consciousness [4].

Epileptic seizures may be part of the clinical condition of encephalopathy [31]. Since we do not record the temporal sequence of neurological symptoms, we are unable to determine whether these epileptic seizures are risk markers for the development of encephalopathy or its symptoms. We are also unable to rule out the fact that some of these patients had encephalitis, although this is rare in COVID-19, since most patients did not undergo imaging tests and none of them underwent a cerebrospinal fluid test.

Older age, smoking, and requiring mechanical ventilation were associated with a prolonged hospitalization. These conditions are associated with more severe forms of COVID-19 $[12,33]$. None of the symptoms or neurological complications was associated with a prolonged length of hospital stay.

Older patients, those requiring mechanical ventilation and those with encephalopathy presented a significantly higher risk of dying. Older age and respiratory failure are wellestablished risk factors for death in COVID-19 [3, 33, 34], Only one previous study has assessed encephalopathy as a risk factor for death and reported the same results as ours [3]. Those with anosmia had a significantly lower risk of death. Our results corroborate what was reported by two other studies $[12,35]$. We consider this to be of clinical importance, since anosmia is an easily measured marker of a good prognosis.

Anosmia occurs more frequently in patients with milder cases of COVID-19 and usually occurs at the onset of the disease, being more associated with viral invasion and the initial response to the virus than with systemic inflammatory conditions $[4,6,11,13]$. Our results probably reflect the pathophysiological process underlying anosmia.

There is controversy in the literature as to whether headache is associated with a better prognosis $[8,9,12]$. However, studies on the subject have either not performed multivariate analysis to control the confounding variables or have not included other neurological symptoms in the analysis. Headache did not prove to be a prognostic marker in our study.

Our study has some limitations. The sample size was not calculated. Thus, we may not have been able to detect small differences. Data were obtained by reviewing medical records. This may have led to underestimating the presence of milder symptoms. The frequency of neurological symptoms is in agreement with that reported by other studies that have used the same methodology as ours, but it is lower than those that directly interviewed patients $[1-5,7,11,12$, 18]. The sample was a convenience sample and the included patients were admitted to hospitals that treat more complex patients, which may have reduced the generalizability of the study.

Our study, however, has a number of strengths. Data were obtained by neurologists and neurology residents. This may have reduced misclassifications. An attempt was made to control for confounding variables related to poor prognosis in the analysis and all neurological symptoms and neurological complications were considered in the prognostic analysis.

\section{Conclusions}

Neurological symptoms are frequent among patients hospitalized with COVID-19. Encephalopathy was the most frequent neurological complication and was associated with older age, diabetes mellitus, previous stroke, a prolonged hospitalization, the presence of epileptic seizures and higher mortality. Those with anosmia had a lower mortality.

Acknowledgements The authors acknowledge Andreia Braga Mota, Eduardo Barbosa Albuquerque Maranhão, Breno José Alencar Pires Barbosa and Marcos Eugenio Ramalho Bezerra for helping in the data collection.

Author contributions PASRF conceived the original idea. PASRF and JEM developed the theoretical framework. Acquisition of data: JEM, DFS, MCS, LMAB, MDPG, and FAAO. Analysis of data: PASRF and 
JEM. Interpretation of the results: PASRF, JEM, DFS, MCS, LMAB, MDPG, and FAAO. All authors discussed the results and contributed to the final version of the manuscript. All authors read and approved the final manuscript.

Funding Not applicable.

Availability of data and materials The data that support the findings of this study are available from the corresponding author upon reasonable request.

\section{Declarations}

Conflict of interest The authors report that there were no conflicts of interest.

Ethics approval The research was approved by the Research Ethics Committees of the hospitals involved. Since data were collected through medical records, the informed consent form was waived by the Ethics Committees.

Consent for publication Not applicable.

\section{References}

1. Karadaş Ö, Öztürk B, Sonkaya AR (2020) A prospective clinical study of detailed neurological manifestations in patients with COVID-19. Neurol Sci 41:1991-1995. https://doi.org/10.1007/ s10072-020-04547-7

2. Liguori C, Pierantozzi M, Spanetta M et al (2020) Subjective neurological symptoms frequently occur in patients with SARSCoV2 infection. Brain Behav Immun 88:11-16. https://doi.org/ 10.1016/j.bbi.2020.05.037

3. Liotta EM, Batra A, Clark JR et al (2020) Frequent neurologic manifestations and encephalopathy-associated morbidity in Covid-19 patients. Ann Clin Transl Neurol 7:2221-2230. https:// doi.org/10.1002/acn3.51210

4. Romero-Sánchez CM, Díaz-Maroto I, Fernández-Díaz E et al (2020) Neurologic manifestations in hospitalized patients with COVID-19: the ALBACOVID registry. Neurology 95:e1060e1070. https://doi.org/10.1212/WNL.0000000000009937

5. Pinna P, Grewal P, Hall JP et al (2020) Neurological manifestations and COVID-19: experiences from a tertiary care center at the Frontline. J Neurol Sci 415:116969. https://doi.org/10.1016/j. jns.2020.116969

6. Saniasiaya J, Islam MA, Abdullah B (2021) Prevalence of olfactory dysfunction in Coronavirus Disease 2019 (COVID-19): a meta-analysis of 27,492 patients. Laryngoscope 131:865-878. https://doi.org/10.1002/lary.29286

7. Vacchiano V, Riguzzi P, Volpi L et al (2020) Early neurological manifestations of hospitalized COVID-19 patients. Neurol Sci 41:2029-2031. https://doi.org/10.1007/s10072-020-04525-z

8. Trigo J, García-Azorín D, Planchuelo-Gómez Á et al (2020) Factors associated with the presence of headache in hospitalized COVID-19 patients and impact on prognosis: a retrospective cohort study. J Headache Pain 21:94. https://doi.org/10.1186/ s10194-020-01165-8

9. Caronna E, Ballvé A, Llauradó A et al (2020) Headache: A striking prodromal and persistent symptom, predictive of COVID-19 clinical evolution. Cephalalgia 40:1410-1421. https://doi.org/10. $1177 / 0333102420965157$
10. Gonzalez-Martinez A, Fanjul V, Ramos C et al (2021) Headache during SARS-CoV-2 infection as an early symptom associated with a more benign course of disease: a case-control study. Eur J Neurol 28:3426-3436. https://doi.org/10.1111/ene.14718

11. Nersesjan V, Amiri M, Lebech AM et al (2021) Central and peripheral nervous system complications of COVID-19: a prospective tertiary center cohort with 3-month follow-up. J Neurol 268:3086-3104. https://doi.org/10.1007/s00415-020-10380-x

12. Amanat M, Rezaei N, Roozbeh M et al (2021) Neurological manifestations as the predictors of severity and mortality in hospitalized individuals with COVID-19: a multicenter prospective clinical study. BMC Neurol 21:116. https://doi.org/10.1186/ s12883-021-02152-5

13. Rocha-Filho PAS, Magalhães JE (2020) Headache associated with COVID-19: frequency, characteristics and association with anosmia and ageusia. Cephalalgia 40:1443-1451. https://doi. org/10.1177/0333102420966770

14. Helms J, Kremer S, Merdji $\mathrm{H}$ et al (2020) Neurologic features in severe SARS-CoV-2 infection. N Engl J Med 382:2268-2270. https://doi.org/10.1056/nejmc2008597

15. Rebora P, Rozzini R, Bianchetti A et al (2021) Delirium in patients with SARS-CoV-2 infection: a multicenter study. J Am Geriatr Soc 69:293-299. https://doi.org/10.1111/jgs.16969

16. Koh JS, De Silva DA, Quek AML et al (2020) Neurology of COVID-19 in Singapore. J Neurol Sci 418:117118. https://doi. org/10.1016/j.jns.2020.117118

17. Taquet M, Geddes JR, Husain M et al (2021) 6-month neurological and psychiatric outcomes in 236379 survivors of COVID19: a retrospective cohort study using electronic health records. Lancet Psychiatry. https://doi.org/10.1016/s2215-0366(21) 00084-5

18. Mao L, Jin H, Wang M et al (2020) Neurologic manifestations of hospitalized patients with Coronavirus Disease 2019 in Wuhan. JAMA Neurol, China. https://doi.org/10.1001/jamaneurol.2020. 1127

19. Lu L, Xiong W, Liu D et al (2020) New onset acute symptomatic seizure and risk factors in coronavirus disease 2019: a retrospective multicenter study. Epilepsia 61:e49-e53. https://doi.org/10. 1111/epi.16524

20. de Oliveira FAA, de Oliveira Filho JRB, Rocha-Filho PAS (2021) Multiple demyelinating sensory and motor mononeuropathy associated with COVID-19: a case report. J Neurovirol. https://doi.org/ 10.1007/s13365-021-01024-5

21. Meppiel E, Peiffer-Smadja N, Maury A et al (2021) Neurologic manifestations associated with COVID-19: a multicentre registry. Clin Microbiol Infect 27:458-466. https://doi.org/10.1016/j.cmi. 2020.11.005

22. de Oliveira FAA, Palmeira DCC, Rocha-Filho PAS (2020) Headache and pleocytosis in CSF associated with COVID-19: case report. Neurol Sci 41:3021-3022. https://doi.org/10.1007/ s10072-020-04694-x

23. de Oliveira FAA, de Melo TFB, Rocha-Filho PAS (2020) Transient lesion in the splenium of the corpus callosum associated with COVID-19. Arq Neuropsiquiatr 78:738

24. de Miranda Henriques-Souza AM, de Melo ACMG, de Aguiar Coelho Silva Madeiro BM et al (2021) Acute disseminated encephalomyelitis in a COVID-19 pediatric patient. Neuroradiology 63:141-145. https://doi.org/10.1007/s00234-020-02571-0

25. Benussi A, Pilotto A, Premi E et al (2020) Clinical characteristics and outcomes of inpatients with neurologic disease and COVID19 in Brescia, Lombardy, Italy. Neurology 95:e910-e920. https:// doi.org/10.1212/WNL.0000000000009848

26. Boldrini M, Canoll PD, Klein RS (2021) How COVID-19 affects the brain. JAMA Psychiat 78:682-683

27. Slooter AJC, Otte WM, Devlin JW et al (2020) Updated nomenclature of delirium and acute encephalopathy: statement of ten 
Societies. Intensive Care Med 46:1020-1022. https://doi.org/10. 1007/s00134-019-05907-4

28. Xiong W, Mu J, Guo J et al (2020) New onset neurologic events in people with COVID-19 in 3 regions in China. Neurology 95:e1479-e1487. https://doi.org/10.1212/WNL.0000000000 010034

29. Nannoni S, de Groot R, Bell S, Markus HS (2021) Stroke in COVID-19: a systematic review and meta-analysis. Int J Stroke 16:137-149

30. Akbar MR, Pranata R, Wibowo A et al (2021) The prognostic value of elevated creatine kinase to predict poor outcome in patients with COVID-19-a systematic review and meta-analysis: creatinine Kinase in COVID-19. Diabetes Metab Syndr Clin Res Rev 15:529-534

31. Garg RK, Paliwal VK, Gupta A (2021) Encephalopathy in patients with COVID-19: a review. J Med Virol 93:206-222

32. Ticinesi A, Cerundolo N, Parise A et al (2020) Delirium in COVID-19: epidemiology and clinical correlations in a large group of patients admitted to an academic hospital.
Aging Clin Exp Res 32:2159-2166. https://doi.org/10.1007/ s40520-020-01699-6

33. Zheng Z, Peng F, Xu B et al (2020) Risk factors of critical \& mortal COVID-19 cases: a systematic literature review and metaanalysis. J Infect 81:e16-e25

34. Li J, Huang DQ, Zou B et al (2021) Epidemiology of COVID-19: a systematic review and meta-analysis of clinical characteristics, risk factors, and outcomes. J Med Virol 93:1449-1458. https:// doi.org/10.1002/jmv.26424

35. Talavera B, García-Azorín D, Martínez-Pías E et al (2020) Anosmia is associated with lower in-hospital mortality in COVID-19. J Neurol Sci. https://doi.org/10.1016/j.jns.2020.117163

Publisher's Note Springer Nature remains neutral with regard to jurisdictional claims in published maps and institutional affiliations. 